\title{
Geometric Hashing and AI-Quantum Deep Learning functional similarities on Remdesivir, drug synergies to treat COVID-19 in Practice.
}

\author{
Ioannis Grigoriadis ${ }^{1}$ \\ ${ }^{1}$ Biogenea Pharmaceuticals Ltd
}

January 14, 2021

\begin{abstract}
Novel SARS coronavirus 2 (SARS-CoV-2) of the family Coronaviridae starting in China and spreading around the world is an enveloped, positive-sense, single-stranded RNA of the genus betacoronavirus encoding the SARS-COV-2 (2019-NCOV, Coronavirus Disease 2019. Remdesivir drug, or GS-5734 lead compound, first described in 2016 as a potential anti-viral agent for Ebola diseade and has also being researched as a potential therapeutic agent against the severe acute respiratory syndrome coronavirus 2 (SARS-CoV-2), the coronavirus that causes coronavirus disease 2019 (COVID-19). Computer-aided drug design (CADD), Structure and Ligand based Drug Repositioning strategies based on parallel docking methodologies have been widely used for both modern drug development and drug repurposing to find effective treatments against this disease. Quantum mechanics, molecular mechanics, molecular dynamics (MD), and combinations have shown superior performance to other drug design approaches providing an unprecedented opportunity in the rational drug development fields and for the developing of innovative drug repositioning methods. We tested 18 phytochemical small molecule libraries and predicted their synergies in COVID-19 (2019- NCOV), to devise therapeutic strategies, repurpose existing ones in order to counteract highly pathogenic SARS-CoV-2 infection. We anticipate that our geometry hashing driven quantum deep learing similarity approaches which is based on separated pairs of short consecutive matching fragments, can be used for the development of anticoronaviral drug combinations in large scale HTS screenings, and to maximize the safety and efficacy of the Remdesivir, Colchicine and Ursolic acid drugs already known to induce synergy with potential therapeutic value or drug repositioning to COVID-19 patients.
\end{abstract}

\section{Hosted file}

BiogenetoligandorolSNNCoMRRS_S_20_029974555.pdf available at https://authorea.com/users/ 389434/articles/504036-geometric-hashing-and-\%CE\%B1\%CE\%B9-quantum-deep-learningfunctional-similarities-on-remdesivir-drug-synergies-to-treat-covid-19-in-practice 